\title{
EL EBRANDING O BRANDING DIGITAL FORTALEZA DEL FUTURO.
}

THE EBRANDING OR DIGITAL BRANDING STRENGTH OF THE FUTURE.

\author{
Arturo Martínez de Escobar Fernández ${ }^{1}$ (D) ; Aránsazu Avalos-Díaz ${ }^{2}$ (DD . \\ 1. Universidad Juárez Autónoma de Tabasco. arturo.martinez@ujat.mx. \\ 2. Universidad Juárez Autónoma de Tabasco. aransazu.avadi@gmail.com. \\ *Correspondencia del Autor: Dr. Arturo Martínez de Escobar Fernández, correo electrónico: arturo.martinez@ujat.mx
}

\section{RESUMEN}

El eBranding o Branding Digital fortaleza del futuro, tiene como principal objetivo, conocer hacia dónde va la comunicación digital, las oportunidades de negocio que supone para las compañías, el rol del eBranding en este nuevo contexto, tiene un enfoque cualitativo con alcance correlacional explicativo, diseño no experimental donde participan las empresas pretendiendo tener una visibilidad en red obteniendo un mayor beneficio para la compañía, satisfaciendo las expectativas de los usuarios cuidando su reputación Online, teniendo como misión traducir el estilo de vida que las marcas, de esta manera, se visualizan los beneficios como una nueva forma de gestión en la utilización de medios digitales, donde se puede ver que se destacan otros contenidos corporativos y alta tecnología por parte del eBranding. Hoy en un mundo sin fronteras, donde la tecnología copia hasta el ADN de las personas, el branding es imprescindible en la gestión de todos los negocios.

Palabras Clave: eBranding digital; imagen; marca; empresas.

Cómo citar:

Martínez de Escobar Fernández, Arturo; Avalos-Díaz, Aránsazu. (2020). El ebranding o branding digital fortaleza del futuro. Revista de Investigaciones Universidad del Quindio, 32(2), 06-15. https://doi.org/10.33975/riuq.vol32n2.447 


\begin{abstract}
ABSTRAC:
The main objective of eBranding or Branding Digital strength of the future, to know where digital communication is going, the business opportunities it entails for companies, the role of eBranding in this new context, has a qualitative approach with explanatory correlate scope, a non-experimental design where companies participate pretending to have network visibility obtaining a greater benefit for the company, meeting the expectations of users taking care of their online reputation, having as their mission to translate the lifestyle that brands, in this way, see the benefits as a new form of management in the use of digital media, where you can see that other corporate and high-tech content is highlighted by eBranding. Today in a world without borders, where technology copies up people's DNA, branding is a must in the management of all businesses.
\end{abstract}

Keywords: Digital eBranding; image; brand; companies.

\section{INTRODUCCION}

"Al ingresar en el siglo XXI, los principales avances en marketing pueden resumirse en un sólo tema: conectividad. Las tecnologías de cómputo, información, comunicación y transporte están transformándose rápidamente y convirtiendo al mundo en un lugar más pequeño. Ahora, más que nunca antes, todos estamos conectados unos a otros y con cosas cercanas y lejanas del mundo que nos rodea. Además, nos estamos conectando de nuevas y diferentes maneras.", (Kotler \& Armstrong, 2008)

En nuestro siglo, el siglo de la globalización, la marca se ha convertido para muchas empresas en su principal patrimonio, su valor en el mercado. Es lo que los consumidores compran y va mucho más allá de la propia materialidad del producto.

Las marcas, a medida que crece la riqueza de los consumidores, se compran porque responden a un determinado estilo de vida. Y es en ese guiño al estilo de vida donde se forma el vínculo marcacliente. La confianza hacia la marca, así como su percepción de calidad,

Aumenta a medida que aumenta la capacidad de la misma de responder al estilo de vida del público al que se dirige, de mimetizar su conducta. En la era de la conectividad, las marcas para mantener su reputación y preservar la confianza que se ha puesto en ellas, deben responder a ese Digital Lifestyle (Sudo, 2012), para posicionarse y consolidarse en el mercado digital donde hoy convergen 4.5 billones de usuarios. (Kemp, 2020)

El eBranding o Branding digital, la creación de imagen de marca digital, tiene como misión traducir el estilo de vida que las marcas evocan en la vida cotidiana a un Digital LifeStyle. el mercado online se ha convertido en parte de la vida cotidiana, en una necesidad imperante para toda la industria publicitaria el orquestar de forma estratégica la comunicación en la red.

El eBranding tiene en este contexto un rol clave a desempeñar: Desde una visión estratégica del mercado digital alineada con el mercado tradicional, unificar criterios de actuación de marca en los distintos canales digitales asentándose sobre un posicionamiento online sólido que transpire los valores de la compañía y proporcione valor al usuario.

Según la Asociación Española de Empresas de Branding "El branding es la gestión inteligente, estratégica y creativa de todos aquellos elementos diferenciadores de la identidad de una marca (tangibles o intangibles). Que contribuyen a la construcción de una promesa y de una experiencia de marca distintiva, relevante, completa $y$ 
sostenible en el tiempo" (AEBRAND, 2021).

Las empresas expuestas a este entorno, desde hoy mismo, desde principios de nuestro siglo, ya tomaron medidas o saben que deben tomarlas para reposicionarse en la red de forma estratégica, dado su impacto no sólo sobre su imagen de marca sino también sobre sus ventas.

El análisis de sitos corporativos y de marca, piezas digitales cuyo objetivo es construir de imagen de marca en la red, ha sido requisito para poder descubrir hacia dónde va la comunicación digital y el rol del eBranding en este nuevo contexto, y debido a lo cambiante del medio, y a su crecimiento rápido, se torna rápidamente obsoleta.

Construir la personalidad de la marca digital siguiendo los patrones de las relaciones humanas es el gran desafío que se le plantea al desarrollo estratégico de la marca digital que a su vez desafía a los profesionales que hoy lideran el mercado de la comunicación interactiva. (Vallet, 2005)

\section{Objetivo del Tema}

Conocer hacia dónde va la comunicación digital, las oportunidades de negocio que supone para las compañías y el rol del eBranding en este nuevo contexto, debido a lo cambiante del medio, y a su crecimiento acelerado, que se torna rápidamente obsoleta.

\section{Importancia}

"El Branding es el proceso de creación, construcción y desarrollo de una marca" "Branding en una organización es todo" (Stalman, ¿Qué es el Branding?, 2015).

El branding, en todas sus formas, proviene del cómo una organización se presenta así misma y a sus productos o servicios y, lo más importante, cómo sus públicos la perciben.

A modo de síntesis podríamos decir sobre el branding:

a. La marca no es el logo. Es completamente diferente de un producto o servicio. Una marca es intangible y existe en la mente del consumidor.

b. Diferentes personas poseen diferentes percepciones, lo que los sitúa en diferentes grados de lealtad hacia la marca.

c. El branding no es garantía al $100 \%$ del éxito en el posicionamiento de una marca en la economía global. Podríamos decir que, aunque se acerca a una ciencia, no es una ciencia exacta.

d. Por propia naturaleza, el branding es estratégico, es la planificación de una serie de actividades que permiten alcanzar un claro objetivo: diferenciar de forma distintiva, singular, a una marca en la mente de las personas.

e. Hoy en un mundo sin fronteras, donde la tecnología copia hasta el $\mathrm{ADN}$ de las personas, el branding es imprescindible en la gestión de todos los negocios, de sus marcas.

Al referirnos al ebranding no nos referimos a la presencia del logo más o menos adaptada a una interfaz digital. Las marcas no son logos. El look and feel o aspecto gráfico de un site o sitio es muy importante, pero sólo es un ingrediente.

\section{Trascendencia}

El Impacto del branding, de la creación y la gestión de marca, en nuestra sociedad ha sido enorme. Cada día, la gente toma conscientemente decisiones para comer, beber, comprar o llevar productos específicos o etiquetas, a menudo sólo por la imagen que proyecta una corporación. Las marcas y el branding nos rodean.

El marketing ha sido el tema más emocionante en los negocios durante las últimas seis décadas, 
dado que es uno de los más cambiantes, este siempre ha girado en torno a la gestión de producto, de cliente y de marcas. Ha venido evolucionando con el transcurrir del tiempo, de la historia y la sociedad. Para los años 50 el marketing estaba enfocado exclusivamente a la gerencia de producto. El denominado marketing 1.0 el cual estaba basado bajo unas directrices tácticas, dado que se buscaba generar demanda.

Según Kotler la crisis estadounidense $\mathrm{y}$ Occidental de los años 60 denominada estanflación (Estancamiento + Inflación), un mal para la economía, pero una bendición camuflada para el marketing (Kotler, 2012) dado que este pasa de ser táctico a estratégico y en algunos casos estratégico superior.

Para las décadas de los setentas y ochentas el marketing pasa del marketing 1.0 al denominado marketing 2.0 el cual se basó en la gestión del cliente, donde el consumidor debería remplazar el producto dentro de las actividades de marketing. En donde se realizaba segmentación, público objetivo y posicionamiento. La llegada del internet y los ordenadores masivos permitieron a las personas tener acceso a la información, generar voz a voz (WOM) y permitieron a los consumidores conectarse entre ellos. Lo cual hace que el concepto del marketing pase a centrase en las emociones humanas, permitiendo la llegada del marketing 3.0. El cual está enfocado al marketing emocional, de experiencias y al valor de marca. El marketing 3.0 nace con el fin de apuntarle al corazón del consumidor, reflejado en la gestión de marca (Kotler, 2012), es la era del marketing horizontal, el cual se encuentra en un entorno cambiante, donde los consumidores confían más entre ellos que en las empresas.

eBranding es sobre toda la experiencia del usuario, es cada aspecto de la relación entre la compañía y el usuario en la red.

Su misión es la de obtener claramente un mayor beneficio para la compañía, satisfaciendo las expectativas de los usuarios. Internet es un medio que se mide, su resultado, el beneficio que aporta a la compañía, es cuantificable. Esta es una de las grandes diferencias entre el eBranding y el Branding, el éxito de la gestión de la marca online se puede medir o cuantificar por:

- Alcance y cobertura: cuántos usuarios hacen clic sobre nuestra marca

- Cuántos usuarios vuelven

- Cuántos usuarios abandonan nuestro sitio web

- Cuánto tiempo están expuestos a nuestra marca

- Qué contenido prefieren

- Qué mensaje ha sido el que más y mejor resultado nos da

- Somos la marca más buscada en los motores de búsqueda con nuestra marca

- ETC.

El eBranding ha de estar entonces presente en toda la cadena del marketing de la empresa, dada su relevancia posee un rol estratégico y por tanto lidera e influye todo proceso de la comunicación digital, y determina la orientación estratégica del marketing interactivo de la empresa.

La relevancia del eBranding estriba en, que hoy posicionar una marca sólo por su valor funcional no funciona. Marcas 100\% nacidas en el mercado online, como por ejemplo Google, empiezan a aplicar eBranding, el de esta era que denominamos de la conectividad. Desde su entrada en el mileno, Google trabaja para añadir valores emocionales que le permitan mantener su relación con los usuarios. El usuario ha cambiado y seguirá cambiando, la red sigue su evolución y las marcas deben evolucionar con ella si desean sobrevivir.

En esta última aserción reside el porqué del eBranding, o te mueves con los valores de la red o te quedas fuera de ella, con todo lo que ello conlleva como es la pérdida de un usuarioconsumidor cuyo perfil representa al prototipo de consumidor por excelencia de nuestra época.

A este porqué eBranding, hemos de añadir: 
a. Estar donde el cliente se informa de la marca y dónde adquirirla

b. Lanzar y testar nuevas marcas o rediseños sin un alto riesgo.

c. Experimentar a bajo costo mercados emergentes, gracias al alto poder de segmentación y de creación de comunidad.

d. Top of mind sostenido. En los años venideros el canal digital será empleado regularmente por la mayoría de los consumidores.

e. Desarrollar fidelidad de marca. Los sitios web nos facilitan el diseñar incentivos a la medida de los usuarios, impulsando visitas susceptibles de transformarse en más ventas.

f. Evitar la destrucción de la marca

g. Aumentar nuestra capacidad de emocionar, haciéndonos más memorables.

h. Sin tener que vender. No hace falta vender en la red, a no ser que sea realmente apropiado para la imagen de marca o para la estrategia de la compañía. emplean la red como un bello escaparate.

i. Hablar cara a cara con el cliente y crecer en credibilidad

j. Reflexionar constantemente sobre el poder de nuestra marca, Internet nos obliga a hacer una readaptación de los atributos de marca.

\section{k. Crear y crecer con el Digital Lifestyle}

\section{Beneficios}

Fue en el año 2004 cuando Tim O'Reilly acuñó el término Web 2.0, refiriéndose a un cambio sufrido en el entorno online. Si algo ha caracterizado a esta nueva web ha sido su manera de fomentar las relaciones sociales y su función colaborativa, llegando así a la creación de comunidades virtuales. Se produjo un crecimiento en el uso de las redes sociales existentes y también surgieron otras nuevas, lo que provocó que el usuario haya dejado de ser un ente pasivo y se implique en la creación de contenidos, así como en la interacción con otros usuarios. Ejemplos de esto son Facebook, YouTube, Twitter, Telegram, entre otros.

Para las marcas esto generó una nueva manera de ver la comunicación con los usuarios; una nueva perspectiva que generaría un afianzamiento de la relación entre la marca y el consumidor. Era el momento de centrarse en las personas, pensar en qué quieren realmente y hacérselo llegar. Al ver esa gran oportunidad que les ofrecía el entorno online, comenzaron a hacerse perfiles en las redes sociales para generar conversaciones con los usuarios mediante su interacción con ellos.

De esa manera podían conocer qué tipo de usuario es, sus gustos, qué le agradaba o disgustaba de la marca... Con esto conseguían, además de una ilimitada cantidad información personal, dar una buena imagen de marca y fomentar el recuerdo de esta, ya que una marca que se comunica con los usuarios es recordada de forma más reciente que otra que no lo hace.

Todo ello (conversaciones, mensajes, interacción, etc.) no es realizado con el objetivo solamente de vender, sino con el de fidelizar. Algunas de las ventajas que se consiguen gracias a la fidelización son la creación de vínculos emocionales con los usuarios, la comunicación boca-oreja que hacen las personas que siguen a una marca y están contentos con ella, la segmentación de los usuarios, la cual permite generar un tipo de comunicación personalizada, o conseguir diferenciarse de demás competidores que ofrecen productos similares.

La idea básica es que es necesario cerrar el círculo, es decir, fomentar el feedback. De esta manera se beneficiará tanto la empresa como el público. La primera podrá fidelizar a los clientes de una manera natural, creando 
lazos de comunidad, a la vez que recibirá una información extraordinariamente valiosa para el éxito de la compañía. El cliente, por su parte, se sentirá realmente escuchado y valorado $\mathrm{y}$, como consecuencia, sus demandas serán satisfechas de una manera más efectiva (Trout et al., 2009).

A los beneficios encontrados en la Web 2.0 se le fueron sumando otros, lo que derivó en la Web 3.0., término que, en este caso, fue utilizado por primera vez en el año 2006 por Jeffrey Zeldman.

La evolución del 2.0 al 3.0 viene dada por los grandes avances hechos en materia de inteligencia artificial, así como en innovación tecnológica, ambos visibles en la web a día de hoy.

La Web 3.0 se caracteriza por varios factores; por su inteligencia en tanto que es capaz de retener los resultados de búsqueda para aplicarlos en futuras transacciones y de realizar búsquedas que se aproximan más al lenguaje coloquial.

La ubicuidad es otro de esos factores ya que, a diferencia de la Web 2.0, los usuarios no solo se conectan a través de un ordenador. Actualmente, cada día son más los que utilizan sus teléfonos móviles inteligentes (smartphones) o tabletas para conectarse a Internet debido a la multifuncionalidad de estos dispositivos, lo que provoca que los usuarios puedan estar conectados en cualquier momento y desde cualquier lugar, no solo desde su casa.

Esto ocurre tanto por el fácil manejo de webs y aplicaciones en dichos dispositivos, como por la rapidez de las comunicaciones y por el auge de las redes sociales, las cuales "obligan" al usuario a estar al día en el perfil que tenga en cada una de ellas y a tenerlas actualizadas en todo momento. También es importante mencionar dentro de estos factores la tendencia cada vez más proclive a la tridimensionalidad, sea en videojuegos o en la manera de navegar por la red, y la utilización de la nube como nueva forma de almacenamiento de la información, dejando atrás antiguos sistemas operativos y guardando la información en el medio online. Google Drive o One drive son ejemplos de la utilización de la Web 3.0.

Las marcas han tenido que acostumbrarse y adaptarse a este nuevo ambiente 3.0. Algunas todavía no juegan en este terreno, pero las que lo hacen (y saben cómo hacerlo) han conseguido muy buenos resultados gracias a la aplicación de nuevas prácticas con los usuarios.

Mostrarse accesible y ofrecer contenido de calidad debería ser una prioridad para las marcas; estar atentos al usuario, resolver dudas rápidamente o difundir buenos contenidos que no tengan que ver explícitamente con vender un producto $\mathrm{o}$ servicio es algo vital para que el usuario entable una relación buena y de confianza con la marca. Estos cada vez exigen más y no se conforman solamente con el producto o servicio ofertado.

Las empresas deben aprender a escuchar, a entender, a mantener una visibilidad en red y a cuidar su reputación online. No cabe duda de que son muchos los desafíos a los que se enfrenta una empresa: adaptarse a la veloz permuta del entorno, cambiar el rol del canal, alinearse con las necesidades del público y ofrecerles mensajes y experiencias valiosas, etc. Pero son también muchos los beneficios y oportunidades que explorar: obtener valor agregado de la inteligencia colectiva, escuchar las necesidades de su público, aprovechar las ideas de los clientes, generar canales más confiables y costeables, etc. Ya no se trata de invertir desorbitadas cantidades de dinero en publicidad u otras tácticas sino de hacerlo bien. Internet permite hacer grandes cosas a costes ridículos.

Lo importante es definir unos objetivos y tener un plan. Por ello, cada vez más los usuarios son implicados en los procesos de creación de las marcas como, por ejemplo, creando un hashtag en Twitter. Se fomenta así la implicación de la marca con el usuario porque esta deja que los usuarios realicen la co-creación de los contenidos, haciéndoles sentir parte de la marca. Todo ello mejora las experiencias del usuario en cuanto al contacto con la marca en el entorno online, 
haciendo que prime lo intangible (satisfacción, sensaciones, experiencia, confianza, etc.) por encima de lo tangible (producto y/o servicio).

Esto ha cobrado tanta importancia que muchas de las marcas ya utilizan intangibles como forma de diferenciación de la competencia.

Se observa de esta manera que la dependencia hacia los medios online ha ido creciendo exponencialmente, sobre todo gracias a la creación de las ya mencionadas redes sociales. Estas son un medio de expresión y comunicación para crear y dar difusión a los contenidos digitales propios o cuya autoría pertenece a otros usuarios. Son, además, un lugar para informarse, comunicar, divertirse y educar. Pero, además, la comunicación comercial publicitaria, corporativa o institucional ha encontrado un espacio, en continuo cambio, en el que experimentar con sus consumidores más allá de la comunicación convencional (Sánchez Hervás, 2013)

Debido a ese espacio que, ha encontrado la comunicación comercial, el branding a nivel online ha comenzado a instaurarse en la vida cotidiana de las marcas. De esta manera, se denomina eBranding a la gestión estratégica de la marca en los medios online, aunque sin dejar de lado al sector offline.

A principio, la mayoría de grandes corporaciones adoptaron entonces una postura conservadora en internet, empleando el canal online principalmente para informar sobre su cartera de productos, sus valores, cultura, personas y su política de relaciones con la prensa, los inversores y otros públicos de su entorno. Dada su naturaleza y misión online como es la creación de imagen corporativa, el site o sitio corporativo podría considerarse como la primera aproximación por parte de la empresa al eBranding (Vallet, 2005)
En el 2001, anunciantes como Coca-Cola son la excepción a la práctica general del mercado y empiezan a despejar dudas sobre qué rol debe cumplir el marketing online y, por ende, la comunicación digital. Coca-Cola con una excelente y agresiva presencia en la red,

Viendo el éxito que se podía alcanzar debido a los medios digitales, el eBranding ha ido cobrando notoriedad. De esta forma, (Vallet, 2005), realizando un estudio tanto de branding como de eBranding, propone una clara diferenciación entre ambos conceptos, plasmando en la siguiente tabla No. 1, las cualidades que los componen:

Tabla No. 1. Diferencias entre branding y eBranding.

\begin{tabular}{|l|l|}
\hline \multicolumn{1}{|c|}{ BRANDING } & \multicolumn{1}{c|}{ EBRANDING } \\
\hline Cualificable & Cuantificable \\
\hline Pasivo & Interactivo \\
\hline Busca & Se Busca \\
\hline $\begin{array}{l}\text { Recuerdo a los 1.200 } \\
\text { GRP's o más }\end{array}$ & Recuerdo al primer clic \\
\hline Estático & Dinámico \\
\hline Sensorial & Multisensorial \\
\hline Esté o no esté & Estés donde estés \\
\hline Inaccesible & Accesible \\
\hline Masivo & Íntimo \\
\hline Estandarizado & Personalizado \\
\hline Difícil de integrar & Fácil de integrar \\
\hline
\end{tabular}

Fuente: (González Reboredo, 2016) basada en el estudio de (Vallet, 2005)

Se puede observar, por lo tanto, los beneficios que aporta el ebranding. Por eso se debe contar con esta nueva forma de gestión, en la utilización de los medios digitales, sobre todo, contando con el desarrollo y avance que ha sufrido hasta llegar a nuestros días. 
Tabla No. 2. Cuadro Comparativo entre Marcas Distinguidas.

\begin{tabular}{|c|c|c|}
\hline Marca & GUCCI & L'ORÉAL \\
\hline Web site & https://www.gucci.com/ & https://www.loreal.com/ \\
\hline $\begin{array}{l}\text { Modelo pure play } \\
\text { site }\end{array}$ & $\begin{array}{l}\text { Tiene un buen impacto visual, para ca- } \\
\text { nalizar a la región a la que te ubicas. } \\
\text { Muy visual, fresca y con lujo. }\end{array}$ & $\begin{array}{l}\text { Tiene un muy buen manejo de medios } \\
\text { visuales, con sobriedad, pero siempre } \\
\text { destacando sus productos. }\end{array}$ \\
\hline Home & $\begin{array}{l}\text { El posicionamiento de lujo, elegan- } \\
\text { cia, frescura y juventud se comunica } \\
\text { óptimamente. Tiene coherencia con la } \\
\text { marca offline. }\end{array}$ & $\begin{array}{l}\text { Apuesta por un formato de portal } \\
\text { para dar la bienvenida, mostrando } \\
\text { de antemano sus productos más } \\
\text { innovadores, con muchos sitios de } \\
\text { interés. }\end{array}$ \\
\hline $\begin{array}{l}\text { Navegación y ar- } \\
\text { quitectura }\end{array}$ & $\begin{array}{l}\text { La barra horizontal de navegación } \\
\text { facilita la navegación rápida por el si- } \\
\text { tio. }\end{array}$ & $\begin{array}{l}\text { Se puede desglosar un menú a la iz- } \\
\text { quierda, el cual muestra en forma } \\
\text { jerárquica la información. }\end{array}$ \\
\hline Diseño & $\begin{array}{l}\text { Imágenes y videos de alta resolución, } \\
\text { y una composición grafica simple log- } \\
\text { rando transmitir los principales atribu- } \\
\text { tos de la marca. }\end{array}$ & $\begin{array}{l}\text { Imágenes y videos de alta resolución, } \\
\text { enfocada a mostrar la más alta tec- } \\
\text { nología e innovación de forma im- } \\
\text { pactante. }\end{array}$ \\
\hline $\begin{array}{l}\text { Contenidos cor- } \\
\text { porativos }\end{array}$ & $\begin{array}{l}\text { Es un site extenso en contenidos cor- } \\
\text { porativos, pero poco profundo, hay } \\
\text { mucha información expuesta al usu- } \\
\text { ario. }\end{array}$ & $\begin{array}{l}\text { Es un site extenso en contenidos cor- } \\
\text { porativos, se destaca por presentar } \\
\text { toda su familia marcas de productos. }\end{array}$ \\
\hline Otros contenidos & $\begin{array}{l}\text { Su contenido es tipo comercial, con un } \\
\text { claro enfoque publicitario. }\end{array}$ & $\begin{array}{l}\text { Destaca en sus contenidos corpora- } \\
\text { tivos y alta tecnología. Actualmente } \\
\text { con mensaje esperanzador por la pan- } \\
\text { demia. }\end{array}$ \\
\hline $\begin{array}{l}\text { UXP, branding y } \\
\text { etapa en internet }\end{array}$ & $\begin{array}{l}\text { Se dedica a informar, destacando a la } \\
\text { vez su marca. No hay interactividad } \\
\text { con la marca, pero deja sus redes so- } \\
\text { ciales para eso. }\end{array}$ & $\begin{array}{l}\text { Busca interacción, tiene un claro } \\
\text { enfoque corporativo y de marketing. }\end{array}$ \\
\hline Redes sociales & $\begin{array}{l}\text { Facebook } \\
\text { Twitter } \\
\text { Instagram } \\
\text { YouTube } \\
\text { Gucci } \\
\text { Podcast } \\
\text { Pinterest } \\
\text { Snapchat }\end{array}$ & $\begin{array}{l}\text { Facebook } \\
\text { Twitter } \\
\text { Instagram } \\
\text { LinkedIn } \\
\text { YouTube }\end{array}$ \\
\hline
\end{tabular}

Fuente: Elaboración Propia.

\section{Caso: Efectos del Sarscov2 en el Branding Digital}

La crisis mundial provocada por el coronavirus ha cambiado los hábitos de consumo y sociales de la población. Con el fin de analizar el impacto de la pandemia en la industria, los negocios y, más concretamente, en el sector del marketing digital, y conocer cómo ha afectado al comportamiento de los consumidores, la agencia publicitaria digital Cool Tabs en colaboración con EUDE Business School es una Escuela de Postgrado especializada 
en la preparación de líderes y profesionales en el ámbito de los negocios realizaron un estudio sobre el impacto que tuvo.

Del estudio se desprende que los hábitos de consumo de la población han cambiado durante la crisis. Así lo refleja el 93\% de los encuestados. Además, más de la mitad, el 63\%, afirma haber comprado alimentos y/o bienes esenciales de forma online durante el período de confinamiento (Cool Tabs \& EUDE Business School, 2020).

Amazon, Mercado libre y Food Delivery App, entre otros han generado un notable crecimiento.

Las marcas han reforzado la comunicación con sus clientes. la mayoría de los encuestados prefiere que las marcas les contacten a través de las redes sociales, o en su misma app, e incluso vía telefónica, frente a otros canales como el email, los SMS u otras aplicaciones.

El confinamiento por el covid-19, sumado a la adopción del teletrabajo como fórmula para mantener la actividad laboral, ha propiciado el desarrollo de nuevas tecnologías, la aplicación de inteligencia artificial y Big Data, así como una obligada transformación digital de numerosas empresas y negocios.

El confinamiento ha provocado un aumento sin igual en el consumo de herramientas $y$ aplicaciones de videoconferencias y reuniones. Tanto es así, que el 94\% de los encuestados (Cool Tabs \& EUDE Business School, 2020) afirma haber utilizado alguna de las aplicaciones de videollamada disponibles como Zoom, Meet, Skype o Teams.

Los consumidores han tenido más tiempo libre y han optado por distintas actividades para no caer en el aburrimiento y la monotonía. Entre estas actividades se encuentran practicar deporte, leer, ver series y películas, y sin duda, una de las que más aclamadas, cocinar. En este sentido, la plataforma de vídeo, Youtube, ha sido la favorita, con un $46 \%$ de respuestas, para seguir recetas y conocer nuevos platos y técnicas culinarias.

La digitalización, clave para la supervivencia de las empresas. La pandemia de covid-19 también ha provocado cambios significativos en la manera de relacionarnos con las marcas y la percepción que los clientes tienen de estas.

la importancia de la digitalización de las empresas. Esta transformación ha sido clave para que muchas empresas y negocios se hayan mantenido a flote durante esta crisis. La digitalización de los procesos empresariales y de las herramientas de trabajo ha sido y seguirá siendo clave durante esta crisis provocada por el covid-19.

La comunicación ha cambiado, el modo de acceder a los productos y servicios y, sobre todo, las expectativas de los clientes hacia las acciones de la marca y la experiencia que deben recibir. Los consumidores son más exigentes y reclaman estrategias en las que el centro sean ellos y se prioricen sus necesidades y deseos.

Aquellas marcas que han sabido adaptarse más rápidamente, han ofrecido servicios extra, más personalizados y han flexibilizado sus medidas son mejor percibidos por los clientes, lo que aumentará su confianza y por ende, su imagen y reputación de marca.

\section{CONCLUSIONES}

El mundo está en constante cambio, y las empresas deben cambiar junto con ellos. Podemos tener un buen producto, una buena marca, pero si realmente no se enfoca en crear una imagen, su propia imagen ante el consumidor (branding digital) y además no interactúa con este (community management) es muy difícil que llegue al éxito.

Las empresas que han sabido prevenir al cambio, y que además aprovecharon un momento de recesión o crisis a nivel mundial como es el caso del SARSCOV2, que han invertido en esta línea del marketing digital, encontraron su nueva 
zona de oportunidad e inclusive les va mejor que antes, y no padecieron crisis económicas, hubo crecimiento económico, y además surgieron nuevas oportunidades de hacer negocio y realzar el valor de una marca.

El sector del Marketing Digital ha sido uno de los fortalecidos durante la crisis en ámbitos como el ecommerce, las redes sociales y el marketing de contenidos. Pese a la reducción, a principios de la crisis, de la inversión publicitaria, poco a poco se recobra la confianza en el mercado y las estrategias digitales son más necesarias que nunca.

Las aplicaciones digitales de videollamada y reuniones han facilitado las comunicaciones entre empresas, empleados y clientes, asimismo han sido una de las vías de conexión más importantes entre la población, no solo en el ámbito laboral, sino también en el personal.

Aquellas marcas que han sabido adaptarse más rápidamente, han ofrecido servicios extra, más personalizados $\mathrm{y}$ han flexibilizado sus medidas son mejor percibidos por los clientes, lo que aumentará su confianza y por ende, su imagen y reputación de marca.

El eBranding tiene en este contexto un rol clave a desempeñar: Desde una visión estratégica del mercado digital alineada con el mercado tradicional.

\section{REFERENCIAS}

1. AEBRAND. (2021). Ebranding. Asociación Española de Empresas de Branding. https://aebrand. org/

2. Cool Tabs, \& EUDE Business School. (2020). La crisis del covid-19 refuerza la relación entre marcas y consumidores. Cool Tabs. https://blog.cool- tabs.com/es/estudio-marketing-digitalcovid-19/

3. González Reboredo, A. (2016). La importancia del eBranding en el posicionamiento de la empresa digital. Análisis de un caso de éxito: la marca Mr. Wonderful. Universidad de Valladolid.

4. Kemp, S. (2020). Digital 2020: 3.8 billion people use social media - We Are Social.

5. We Are Social. https://wearesocial.com/blog/2020/01/digital-2020-3-8-billion- people-use-socialmedia

6. Kotler, P., \& Armstrong, G. (2008). FUNDAMENTOS DE MARKETING (Pearson \& Prentice Hall (eds.); 8va ed.).

7. Sánchez Hervás, D. (2013). Estrategias de comunicación en redes sociales.

8. Usuarios, aplicaciones y contenidos. Sphera Pública. Universidad Católica San Antonio de Murcia España, 1, 183-185. http://www.redalyc.org/articulo.oa?id=29732391011

9. Sudo, L. (2012). In the world of social media, what's your digital lifestyle? Communicatto. Https://Www.Communicatto.Com/. https://www.communicatto.com/blog/in-the-world-ofsocial-media-whats-your- digital-lifestyle/

10. Trout, J., Rivkin, S., \& Peralba, R. (2009). Diferenciarse o morir: cómo sobrevivir en un entorno hipercompetitivo de alto riesgo (Pirámide (ed.); 2nd ed.).

11. Vallet, G. (2005). eBranding. La creación de la marca digital en la era de la conectividad. In Universidad Autónoma de Barcelona. https://www.tdx.cat/handle/10803/4125\#page=1 